\title{
GPU-Accelerated Molecular Visualization on Petascale Supercomputing Platforms
}

\author{
John E. Stone \\ Beckman Institute \\ University of Illinois \\ 405 N. Mathews Ave. \\ Urbana, IL 61801 \\ johns@ks.uiuc.edu
}

\author{
Kirby L. Vandivort \\ Beckman Institute \\ University of Illinois \\ 405 N. Mathews Ave. \\ Urbana, IL 61801 \\ kvandivo@ks.uiuc.edu
}

\author{
Klaus Schulten \\ Department of Physics \\ University of Illinois \\ 1110 W. Green \\ Urbana, IL 61801 \\ kschulte@ks.uiuc.edu
}

\begin{abstract}
Petascale supercomputers create new opportunities for the study of the structure and function of large biomolecular complexes such as viruses and photosynthetic organelles, permitting all-atom molecular dynamics simulations of tens to hundreds of millions of atoms. Together with simulation and analysis, visualization provides researchers with a powerful "computational microscope". Petascale molecular dynamics simulations produce tens to hundreds of terabytes of data that can be impractical to transfer to remote facilities, making it necessary to perform visualization and analysis tasks in-place on the supercomputer where the data are generated. We describe the adaptation of key visualization features of VMD, a widely used molecular visualization and analysis tool, for GPU-accelerated petascale computers. We discuss early experiences adapting ray tracing algorithms for GPUs, and compare rendering performance for recent petascale molecular simulation test cases on Cray XE6 (CPUonly) and XK7 (GPU-accelerated) compute nodes. Finally, we highlight opportunities for further algorithmic improvements and optimizations.
\end{abstract}

\section{Categories and Subject Descriptors}

I.6.8 [Simulation and Modeling]: Types of SimulationParallel; I.3.7 [Computer Graphics]: Three-Dimensional Graphics and Realism; D.1.3 [Concurrent Programming]: Parallel programming

\section{General Terms}

Algorithms, Design, Performance

\section{Keywords}

Molecular visualization, molecular surface, GPU, ray tracing, parallel rendering, many-core

Permission to make digital or hard copies of all or part of this work for personal or classroom use is granted without fee provided that copies are not made or distributed for profit or commercial advantage and that copies bear this notice and the full citation on the first page. Copyrights for components of this work owned by others than ACM must be honored. Abstracting with credit is permitted. To copy otherwise, or republish, to post on servers or to redistribute to lists, requires prior specific permission and/or a fee. Request permissions from Permissions@acm.org.

UltraVis'13 November 17, 2013, Denver, CO, USA

Copyright 2013 ACM 978-1-4503-2500-4/13/11...\$15.00.

http://dx.doi.org/10.1145/2535571.2535595 .

\section{INTRODUCTION}

Molecular dynamics simulations provide researchers with a "computational microscope", a powerful tool that provides dynamic views of cellular processes with atomic detail and nanosecond temporal resolution that can not be reached by experimental methods alone. Indeed, key discoveries have been made with the computational microscope alone [10]. Petascale supercomputers extend the reach of the computational microscope to very large biomolecular systems that are actually of particular interest to public health, e.g., in the case of viruses such as HIV [27]. Visualization of the dynamics of such large biomolecular systems assists researchers in their efforts to devise new strategies for treatment, for example to explore new drug treatment.

The creation of informative and compelling visualization from terabytes of simulation data raises the issue of where best to perform visualization [26] and requires close coupling of visualization and analytical functionality, and userextensibility, key features of the molecular visualization and analysis package VMD [6]. VMD was one of the first molecular visualization tools to employ Graphics Processing Units (GPUs) for functions beyond OpenGL rasterization [22]. Led by VMD's broad use of GPUs, the molecular modeling field has subsequently seen broad adoption of GPUs for many computationally demanding algorithms [19]. The increasing popularity of GPUs for acceleration of scientific codes, and the benefits they bring to energy efficiency [5] have recently motivated the incorporation of GPUs in several of the fastest supercomputers in the world, including Titan at Oak Ridge National Laboratory and Blue Waters at the National Center for Supercomputing Applications at the University of Illinois. This incorporation has created many new opportunities for in-place visualization, further enhancing the benefits of this approach and eliminating some of the drawbacks previously associated with visualization on high-end supercomputing systems.

Over the past five years VMD has been adapted to leverage the computational capabilities of GPUs for acceleration of data-parallel algorithms, initially focusing on the computationally most demanding non-graphical algorithms such as calculation of electrostatic potential maps and molecular dynamics simulation trajectory analysis routines $[5,11]$. The incorporation of GPUs into petascale computing systems has created a new opportunity to apply GPU acceleration to the creation of advanced visualizations and movie renderings. Over the past year VMD has been adapted for execu- 
tion on petascale systems using a hybrid computational approach that combines distributed memory message passing with MPI, shared memory multithreading, and GPU acceleration [20]. We describe the ongoing adaptation of VMD to exploit the unique visualization capabilities of the Cray XK7 GPU compute nodes, including GPU-accelerated algorithms for molecular surface visualization adapted to handle very large biomolecular complexes, and a new GPU-accelerated ray tracing engine capable of reproducing complex shadowing and lighting effects not feasible in interactive visualization tools or rasterization APIs such as OpenGL. The new GPU-accelerated ray tracing engine provides a low-overhead high-performance mechanism for rendering molecular graphics, taking advantage of direct access to internal VMD scene data structures and providing direct support for rendering of spheres and other curved geometric primitives, thereby reducing memory requirements. We evaluate the benefits of our GPU-accelerated molecular visualization techniques on the Blue Waters Cray XK6/XK7 petascale system. The algorithms and techniques we describe are also applicable to smaller GPU-accelerated clusters and personal computers.

\section{RELATED WORK}

The most widely used molecular surface algorithm is the Solvent Excluded Surface (SES) [2, 16, 24], determined by the contact surface resulting from rolling a spherical probe over all atoms. The computation of SES is currently prohibitive for large molecular complexes due to computational complexity and memory requirements. Even recent, parallel methods $[8,12]$ can only render the SES for data sets of less than $2 \mathrm{M}$ atoms on current GPU hardware (SES memory requirements can exceed $1.4 \mathrm{~GB}$ for an input set of only 180,000 atoms [4]). D'Agostino [3] has presented a method for using CUDA to generate molecular surfaces (using the Connolly method [2]) which provides a speedup of $88 \times$ for a data set of 90,898 atoms (the largest system for which results were provided) with a grid spacing of $0.5 \AA$. Surface methods such as SES that are focused on reproducing the fine details of solvent-accessibility are largely complementary to our approach, which handles multi-million atom complexes.

Blinn [1] introduced another molecular surface algorithm based on summation of Gaussian density functions, which is the approach taken by our algorithm. Molecular surfaces can be rendered directly from volumetric density data using ray casting techniques, but the memory capacity limitations of current GPU hardware limit ray casting approaches to small molecular structures [7, 13], or require densities to be evaluated on-the-fly as part of rendering [15]. Our approach extracts triangle mesh isosurfaces from computed densities, accommodating very large molecular complexes using a multipass approach. The use of a pre-computed isosurface mesh during ray tracing enables rendering of molecular complexes containing tens to hundreds of millions of atoms, and avoids recomputation of densities during ray tracing of secondary rays for transmission rays, shadows, and ambient occlusion lighting effects, and when rendering stereoscopic views or movie sequences where only the camera orientation changes.

\section{SOFTWARE ARCHITECTURE}

The visualization features of VMD act as a pipeline that transforms the low-level atomic structure information into molecular graphics that show the key structural details se-

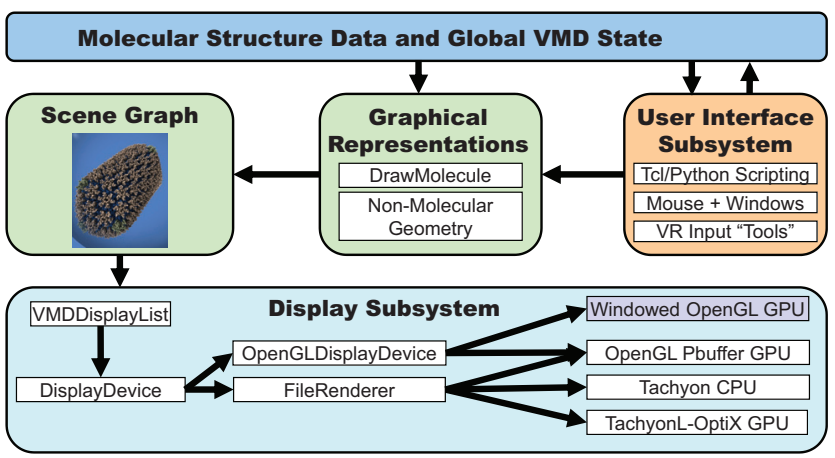

Figure 1: Flow of information through VMD software subsystems during the rendering process.

lected by a user. The user defines a set of so-called graphical representations, each of which combine an atom selection that specifies the portion of the molecular structure to be drawn with a drawing method, a coloring method, a material that controls lighting and shading properties, and various settings for temporal averaging and filtering of atomic coordinates, superposition of multiple trajectory timesteps, and display of additional periodic unit cell instances.

VMD was originally developed for the CAVE and other immersive environments, but subsequently became a widely used desktop application [6,21]. The necessity to support a diversity of display hardware led to a $\mathrm{C}++$ class structure with a DisplayDevice base class that abstracts details of the graphics system and makes it possible to support a broad range of internal and external renderers and scene file formats through subclassing. In the context of batch mode visualization on supercomputers, the DisplayDevice subclasses for OpenGL Pbuffer rasterization, Tachyon CPU ray tracing, and TachyonL-OptiX GPU ray tracing are most relevant, as illustrated in Fig. 1.

Since VMD was originally designed for interactive display of molecular graphics, the data structures and memory layout of geometric information in the scene graph use inmemory storage formats supported by OpenGL APIs. While most graphics libraries and scene file formats share many data organization principles, the exact in-memory storage schemes used by different packages tend to be incompatible, requiring DisplayDevice subclasses to convert the OpenGLcentric VMD memory layouts to those required by target renderer. By implementing fully-customized virtual methods for a larger fraction of the geometric primitives that VMD uses and by optimizing color and material state changes for the target renderer, a DisplayDevice subclass can achieve higher quality output, greater memory or disk storage efficiency, or greater performance through optimal handling of geometric primitives. It is often particularly beneficial to performance for DisplayDevice subclasses to provide optimized primitives for curved surfaces such as spheres or other quadric surfaces, rather than planar faceted triangle meshes, and for efficient handling of large arrays of primitives.

\section{CRAY XE6/XK7 OVERVIEW}

Blue Waters is a hybrid of Cray XE6 (CPU-only) and XK7 (hybrid GPU-accelerated) compute nodes attached to a high performance Cray Gemini network and Sonexion storage systems based on the Lustre parallel filesystem. The machine currently consists of 237 Cray XE6 cabinets $(45,280$ 
AMD Opteron 6276 16-core CPUs achieving 7.1 petaflops peak), and 44 XK7 cabinets (4,220 AMD Opteron 6276 CPUs and 4,220 NVIDIA Tesla K20X GPUs achieving 6.2 petaflops peak). Blue Waters nodes utilize AMD Opteron "Interlagos" 6276 CPUs, each with 16 integer cores sharing 8 floating point units. The XE6 nodes contain two CPUs and $64 \mathrm{~GB}$ of RAM. The XK7 nodes contain one CPU and $32 \mathrm{~GB}$ of RAM, and an attached NVIDIA Tesla K20X "Kepler" GPU accelerator with 6 GB of RAM.

The XK7 Tesla K20X GPUs are composed of 14 "SMX" SIMT execution units that contain hundreds of individual single- and double-precision arithmetic units, special function units, and load/store units; SMXs are capable of simultaneously scheduling independent instructions on different units. The modest size of the $6 \mathrm{~GB}$ on-board GPU memory implies that for many molecular analysis and visualization calculations only a small subset of the molecular data structures can reside on the GPU, and they may have to be evicted on-demand in order for subsequent calculations to fit within the GPU memory. GPU memory capacity is a critical issue when working with petascale molecular dynamics trajectories produced on leadership class systems, and it must be directly addressed in the design of GPU algorithms. The Blue Waters XK7 GPU system software also supports hardware-accelerated OpenGL rasterization, presently a unique feature among petascale computers [20].

\section{MOLECULAR REPRESENTATIONS}

The graphical representations created by VMD from molecular structure information are implemented internally by $\mathrm{C}++$ classes and methods that traverse lists of selected atoms, accessing their properties such as atomic coordinates, radii, atomic element, structural component tags, and so on, to produce lists of low-level geometric primitives that are then inserted into the VMD scene graph. VMD contains algorithms for generating the most widely used molecular graphics representations and the computational demands made by these algorithms scale with the size of the molecular system (i.e. with the total atom or particle count).

Graphical representations such as molecular orbitals, molecular surfaces, and isosurfaces of various types are computationally much more demanding than other representations due to a combination of arithmetic- and/or memory-intensive algorithm steps and in some cases due to greater algorithmic time complexity, making them compelling targets for GPU acceleration techniques. The key issues for determination of the feasibility of GPU algorithms for a particular molecular representation hinge upon the need for large amounts of independent work required for development of data-parallel algorithms, and a sufficient number of work units to fully hide GPU instruction scheduling and memory latencies.

Molecular surface representations have proven to be particularly useful for visualization of large biomolecular complexes such as viruses, photosynthetic organelles, and cell membranes, and also for synthetic silicon nanodevice simulations. There are many molecular surface definitions and a wide variety of approaches for computing and visualizing them efficiently under various assumptions. For visualizing very large molecular complexes, molecular surface definitions and surface determination algorithms that can handle very large particle counts and provide surfaces with user-specified detail are most interesting. Solvent accessible surface generation approaches, which are constrained to

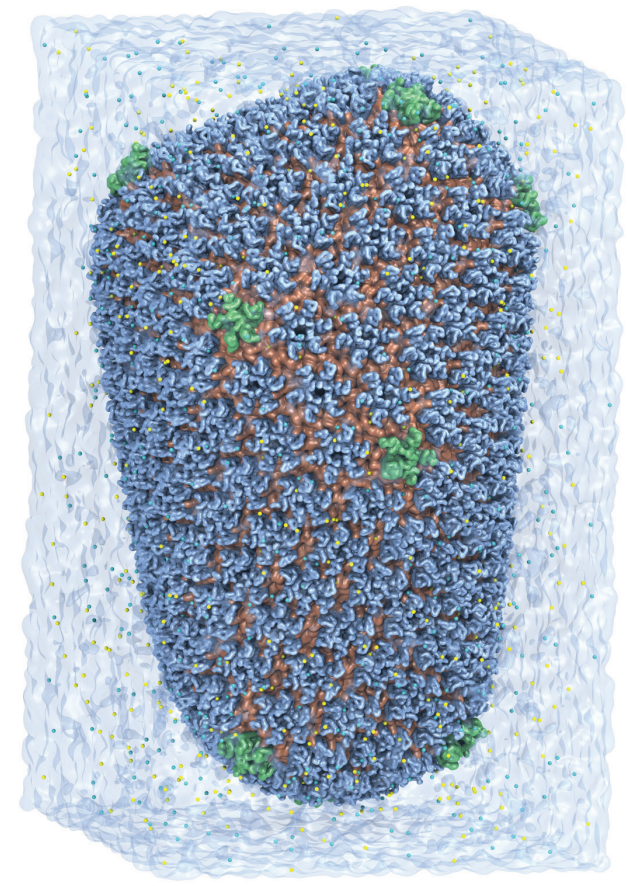

Figure 2: VMD "TachyonL-OptiX" overview rendering of the 64-million-atom simulation of the HIV1 capsid [27] from the HIV movie used for performance testing. This view shows the extent of the molecular dynamics simulation unit cell with solvent, ions, and multiple surface representations highlighting components of the HIV-1 capsid. The rendered scene includes ambient occlusion lighting, transparent surfaces, transmission rays, and filtering of shadows by transparent surfaces, and multiple geometric primitives consisting of triangle meshes, sphere arrays, and cylinder arrays.

operating at full atomic detail or that have memory requirements that grow rapidly with increasing atom counts, are valuable tools in the context of illustrating small regions of a molecular complex in great detail, but their applicability for visualizing cellular organelles or biomolecular complexes containing millions of atoms or other particles is therefore very limited.

We have previously developed a molecular surface rendering algorithm based on generation of a synthetic density map created by summation of Gaussian densities followed by isosurface extraction. The surface algorithm is related to Blinn's approach [1] and employs a spatial acceleration data structure along with a distance-limited density summation approach to achieve linear time-complexity with the number of particles. The surface algorithm also includes the ability to reduce computational demands associated with large particle counts through per-residue "bead" simplification [9]. This surface algorithm focused on achieving interactive surface generation rates and OpenGL rendering speeds on molecular structures of up to a few million atoms, using GPU-accelerated density map synthesis and isosurface extraction stages. The surface algorithm has been enhanced subsequently with support for cellular scale structures that incorporate a very large range of particle sizes, and with a multi-core CPU implementation capable of handling hun- 


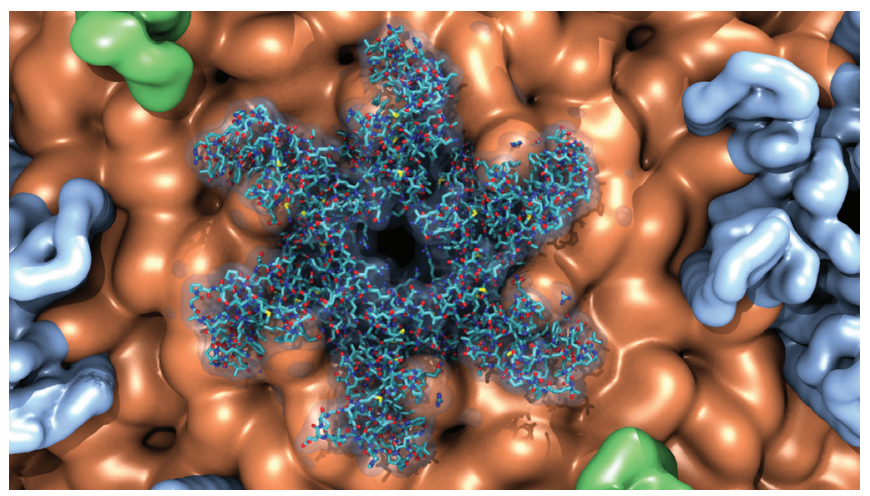

Figure 3: VMD "TachyonL-OptiX" close-up rendering of the all-atom atomic structure of the HIV-1 capsid [27] taken from the HIV sequence used for performance testing.

dreds of millions of particles at non-interactive rates [17].

The present work extends the GPU-accelerated surface algorithm QuickSurf [9] to very large molecular complexes that exceeded on-board GPU memory capacities when using the previous algorithm, and adds hand-coded SSE vectorization to the multi-core CPU code path. The images shown in Figs. 2, 3, 5 each make use of surface representations that border on the limits of GPU on-board memory capacity. An additional constraint of the current work is that the synthetic density map algorithm is used not only for visualization purposes, but is also used as the basis for GPUaccelerated algorithms in a new GPU-accelerated version of the Molecular Dynamics Flexible Fitting (MDFF) method under development [25]; changes to the density map implementation had to be free of deleterious consequences to the performance or accuracy of those algorithms.

The first step in enhancing the QuickSurf surface algorithm for large structures involved further enhancement and hardening of the code against various kinds of GPU outof-memory conditions with extensive checking, and by performing all memory allocations for all algorithm phases together in unison prior to the beginning of the calculation. This prevents unexpected memory problems during surface generation, and allows the algorithm to handle memory exhaustion scenarios, either by performing the surface calculation in multiple passes, or, in the worst case, by falling out of the GPU code path and returning to the multi-core CPU code path. The enhanced multi-pass surface calculation illustrated in Fig. 4 decomposes the volume containing the set of selected atoms or particles into "slabs", which are a multiple of 8 density map lattice points in each dimension. The $8 \times 8 \times 8$ density map block size maps directly to the size of a CUDA thread block on the GPU, and also takes into account the number of density map lattice points computed within each GPU thread using loop unrolling and register tiling techniques. As the density map generation algorithm processes all of the slabs within the volume of interest, it must save the last plane of the previous slab so that the subsequent marching cubes isosurface extraction phases generate surfaces that connect with the previous batch of computed triangles.

Unlike our previous work where the triangle meshes produced by individual slab iterations were rasterized by OpenGL on-the-fly as they were produced [9], the approach described

\section{Density Map Parallel Decomposition}

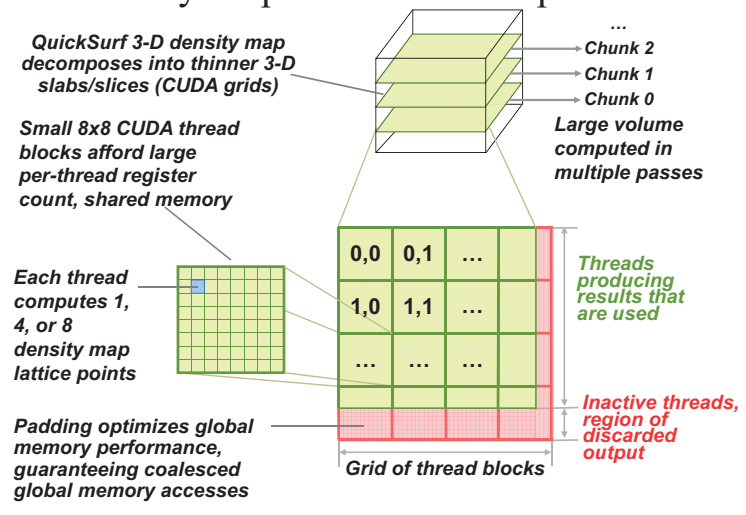

Figure 4: Overview of multi-pass surface generation and parallel decomposition. Density slab computation and surface extraction are performed entirely on the GPU, but due to memory capacity limitations, the resulting triangle mesh is transferred back to the host. The larger Cray XK7 host memory capacity allows the final surface geometry to be stored for rendering at a later time. The entire scene must be retained for ray tracing, as opposed to the streaming approach used for OpenGL rasterization.

here stores all of the triangles produced from the isosurface extraction step back to host memory so that the entire molecular scene can be presented to the rendering engine at once, thereby avoiding costly recalculation at render time, which would be inefficient for very large surfaces. The hostbased storage of the final triangle mesh, while not optimal for the case of interactive OpenGL rasterization, allows all on-board GPU memory used during surface generation to be completely deallocated, making all of the GPU memory resources available for GPU-accelerated ray tracing.

The second modification made to the QuickSurf surface generation algorithm involved a redesign of both the density map kernels and the marching cubes implementation to support multiple data types for density values, color values, and surface normals. The availability of $\mathrm{C}++$ style polymorphism, operator overloading, and templates in recent CUDA versions has made it possible to redesign the density map and marching cubes algorithms in a substantially more type-generic way, with an almost unmeasurable cost to performance due to special properties of the algorithm.

The new type-generic version of the density map algorithm uses $\mathrm{C}++$ templates to cause the CUDA compiler to generate a type-specific version of the main density map kernel, replacing the previous hard-coded float single-precision floating point density map type and the CUDA float3 3-D texture map color type with template-instantiated DENSITY and VOLTEX types which are automatically resolved at compiletime. The internal density and color determination in the kernel is still performed using single-precision arithmetic, but the final in-register results are converted to the templateinstantiated types immediately prior to storing them to GPU global memory. This avoids the complexity and overhead of performing type conversions more frequently inside the density map loops, and the impact to overall kernel design is minimal because it is a so-called gather style parallel algorithm that builds values primarily in on-chip registers. This 
low cost of performing a type conversion operation prior to storing register values to GPU global memory is a significant advantage for gather style algorithms. Matching changes were made to the structure of the GPU marching cubes isosurface extraction algorithm, enabling a broad range of compile-time density, color, and surface normal memory representations to be evaluated in terms of memory consumption, performance gain or loss, and impact on the quality of the final surface renderings.

After trying several different combinations of data types that included single-precision floating point words, the socalled half precision 16-bit floating point type (supported by CUDA and other graphics APIs), unsigned character representations for colors and signed character representations for surface normals, the best combination of performance, memory efficiency, and final surface quality in our testing has been achieved with the use of float single-precision density values and vertex coordinates, uchar4 4-element unsigned character vectors for colors, and char3 signed character surface normals. We used the quantization approach described in Table 2.6 of the OpenGL specification version 1.2.1 for our implementation of signed-character surface normal arithmetic, to allow the final triangle meshes to be used both by our custom-written ray tracers as well as for OpenGL rasterization.

State-of-the-art GPUs are capable of performing up to 40 arithmetic operations per global memory reference, making memory bandwidth a primary consideration for most GPU algorithms. The new type-optimized surface algorithm performs up to $2 \times$ faster than the previous algorithm when rendering surfaces with independent coloring properties applied to each particle. When rendering single color surfaces (one color for the entire set of triangle meshes), the performance of the new algorithm is roughly $1.5 \times$ faster. The performance gains with the new surface algorithm are the direct result of the decreased GPU global memory bandwidth required when writing in-register density and color results to global memory, followed by density and color reads performed within the marching cubes isosurface extraction.

\section{GPU RAY TRACING}

As described above in the discussion of the software architecture of VMD, VMD incorporates multiple DisplayDevice and FileRenderer subclasses that implement rendering techniques for high-performance, high-quality molecular visualizations. In particular, VMD incorporates renderers for both GPU-accelerated OpenGL rasterization, and photorealistic lighting and publication-quality rendering using parallel ray tracing techniques. One of the principal achievements in the current version of VMD is implementation of a new GPU-accelerated ray tracing engine, which outperforms the previous multi-core CPU implementation. The ability to perform large scale rendering jobs directly on petascale supercomputers allows users to avoid the transfer of large simulation trajectories to other sites for rendering. The high performance parallel movie rendering infrastructure in VMD allows rapid completion of photorealistic movie rendering that would take weeks or months (even with the acceleration provided by GPUs) on a desktop workstation [20].

VMD has used a built-in library version of the Tachyon [18] parallel ray tracing engine since 2002. Tachyon provides a lightweight software API that is easy to integrate, and, as a result of a decade of ongoing co-development with VMD,

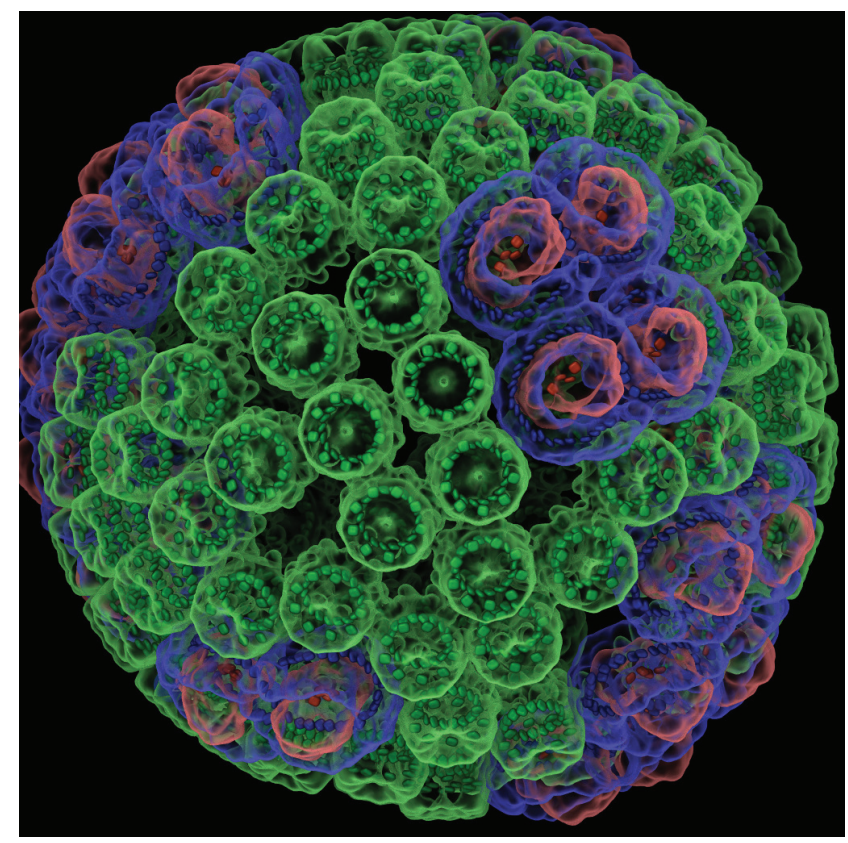

Figure 5: VMD "TachyonL-OptiX" rendering of a chromatophore structure [23], incorporating ambient occlusion lighting, angle-dependent transparency, depth cueing, and transmission rays with deep ray recursion.

supports many rendering primitives, data types, and triangle mesh formats required for efficient rendering of molecular scenes produced by VMD. We have previously reported early results running the CPU-based versions of Tachyon on the Blue Waters Cray XE6/XK7 petascale computer, outlining performance optimizations and work-scheduling improvements that benefit both intra-node multi-core rendering performance as applied to VMD movie rendering jobs on large node counts [20].

The present work describes a redesign of key parts of the Tachyon ray tracer required by VMD for GPU-accelerated execution on both desktop workstations and, particularly, for GPU-accelerated petascale computing systems. The existing CPU-based version of Tachyon is written in ANSI C using a hand-tuned object-oriented structure with manually constructed virtual method tables similar to what a $\mathrm{C}++$ compiler would generate, but that are optimized for ray tracing. The design of Tachyon internal data structures preceded the widespread availability of SIMD floatingpoint arithmetic and is written using a traditional array-ofstructures memory layout. Tachyon currently relies entirely on compiler autovectorization (with limited success) to exploit CPU SIMD units.

GPU hardware is composed of a large number of multithreaded SIMD arithmetic units (sometimes described as single-instruction multiple-thread, or "SIMT"), which favor structure-of-arrays memory layouts and execution of wide stripes (warps) of data-parallel threads or work-items in lock-step. The differences in low-level architecture make the process of porting an existing object-oriented ray tracing engine to the GPU difficult, even for expert GPU programmers, due to the complexities of maintaining sufficient numbers of active rays to feed the SIMD arithmetic units, han- 


\begin{tabular}{|c|c|c|c|}
\hline Node Type & $\begin{array}{c}\text { Loading, } \\
\text { Geometry } \\
\text { Calc. }\end{array}$ & $\begin{array}{c}\text { TachyonL- } \\
\text { OptiX } \\
\text { GPU } \\
\text { Rendering }\end{array}$ & $\begin{array}{c}\text { Tachyon } \\
\text { CPU } \\
\text { Rendering }\end{array}$ \\
\hline$\overline{\text { XK7 }}$ & $27 \mathrm{~s}$ & $145 \mathrm{~s}$ & \multirow{2}{*}{$2,448 \mathrm{~s}$} \\
\hline XK7/No GPU & $257 \mathrm{~s}$ & $\mathrm{n} / \mathrm{a}$ & \\
\hline XE6 & $260 \mathrm{~s}$ & $\mathrm{n} / \mathrm{a}$ & $1,906 \mathrm{~s}$ \\
\hline Xeon E5 GPU & $16 \mathrm{~s}$ & $91 \mathrm{~s}$ & \multirow{2}{*}{$984 \mathrm{~s}$} \\
\hline Xeon E5 & $127 \mathrm{~s}$ & $\mathrm{n} / \mathrm{a}$ & \\
\hline $\begin{array}{l}\text { Xeon X5550 } \\
\text { Two GPUs }\end{array}$ & $22 \mathrm{~s}$ & $132 \mathrm{~s}$ & \multirow[t]{2}{*}{$2,163 \mathrm{~s}$} \\
\hline Xeon X5550 & $148 \mathrm{~s}$ & $\mathrm{n} / \mathrm{a}$ & \\
\hline
\end{tabular}

Table 1: Single Frame Rendering Performance.

dling divergent execution, and GPU hardware architectural limitations that remain necessary even with state-of-the-art GPUs in order to maintain high performance.

To facilitate the development of GPU ray tracing engines, NVIDIA has released the OptiX ray tracing toolkit, which provides the fundamental components for scheduling ray intersection tests, managing the mapping of work to SIMD units, and coping with various hardware limits [14]. OptiX internally implements several high performance GPUoptimized spatial acceleration data structures based on bounding volume hierarchies (Bvh) and eliminates the need for developers to implement the routines themselves. The remaining components of a traditional ray tracing system, including intersection test routines, bounding volume determination, geometry data structures, material property handling, lighting calculations, and shading algorithms, are all written by the developer, and are written as GPU-native CUDA device functions that are compiled to PTX intermediate code (a pseudo machine language for NVIDIA GPUs) with the NVIDIA CUDA compiler tools. At runtime, the main entry point functions are specified by the calling application, and the OptiX runtime system reads the previously-compiled PTX intermediate code and builds the complete ray tracing engine by combining its internal work scheduling and acceleration structure code with the caller-provided intermediate code and prepares it for execution.

We implemented a new GPU-accelerated ray tracing engine in VMD by rewriting the existing Tachyon multi-core CPU ray tracer into a set of CUDA device functions that are loaded at runtime into the OptiX framework, forming the complete ray tracing engine referred to here as "TachyonLOptiX". The core of the Tachyon CPU ray tracing engine in VMD is composed of over 14,000 lines of C source code. The newly developed TachyonL-OptiX engine contains roughly 4,000 lines of more concise CUDA $\mathrm{C}$ and $\mathrm{C}++$ code. The geometric primitives and shading features implemented in TachyonL-OptiX are currently a subset of what Tachyon provides, prioritized by the needs of VMD and against alternatives that are better-suited to GPU hardware. One of the key differences between the original Tachyon and TachyonLOptiX is that peak GPU performance revolves around the use of structure-of-array memory organizations and the effective use of power-of-two size vector types, e.g., float1, float2, float4. In cases where the most convenient memory layout for a geometric primitive happened to agree with the GPU memory layout best practices, the natural organization of data in array-of-structure format was preserved.

In deciding which geometric primitives to implement in

\begin{tabular}{|c|c|c|c|c|}
\hline $\begin{array}{l}\text { Samples } \\
\text { Per-pixel }\end{array}$ & Shadows & $\begin{array}{l}\text { Amb. } \\
\text { Occ. }\end{array}$ & $\begin{array}{c}\text { Ray } \\
\text { Depth }\end{array}$ & Time \\
\hline 1 & off & off & $\begin{array}{c}1 \\
10 \\
20\end{array}$ & $\begin{array}{l}0.065 \mathrm{~s} \\
0.191 \mathrm{~s} \\
0.195 \mathrm{~s}\end{array}$ \\
\hline 12 & off & off & $\begin{array}{c}1 \\
10 \\
20\end{array}$ & $\begin{array}{l}0.416 \mathrm{~s} \\
1.231 \mathrm{~s} \\
1.260 \mathrm{~s}\end{array}$ \\
\hline 12 & on & off & $\begin{array}{c}1 \\
10 \\
20\end{array}$ & $\begin{array}{l}2.066 \mathrm{~s} \\
7.374 \mathrm{~s} \\
7.606 \mathrm{~s}\end{array}$ \\
\hline 144 & on & on & $\begin{array}{c}1 \\
10 \\
20\end{array}$ & $\begin{array}{l}41.737 \mathrm{~s} \\
152.29 \mathrm{~s} \\
156.22 \mathrm{~s}\end{array}$ \\
\hline
\end{tabular}

Table 2: Single Frame TachyonL-OptiX Chromatophore Ray Tracing Time.

TachyonL-OptiX, we evaluated several trade-offs between the potential performance impact of execution divergence, because rays must be intersected with a wider variety of geometric primitive types, but the SIMD hardware requires lock-step execution of threads within SIMD warps. We chose to implement sphere arrays, cylinder arrays, and several triangle mesh formats, including variants that employ the reduced-precision color and surface normal scheme for molecular surface representations described above. The implementation of custom primitives for curved geometry makes efficient use of GPU memory systems because several hundred triangles are required to provide a useful visual approximation of a sphere. The reduction in the total number of objects from direct implementation of curved geometric primitives also benefits the performance of the OptiX Bvh spatial subdivision acceleration algorithms.

One issue that arose in rewriting Tachyon for the GPU was the limitation that GPU kernels are allowed to run only for a brief period of time before a so-called "watchdog" timer in the host operating system terminates the running kernel. This behavior is required by most windowing systems and arises on Blue Waters as a result of its support for off-screen OpenGL Pbuffer rendering [20]. In practice, this prevents ray tracing kernels from being written traditionally with significant internal looping for shooting shadow test rays, ambient occlusion samples, transmission rays or reflection rays, because the runtime for a ray launch can exceed the timeout limit. Avoiding this problem required that looping be implemented in large part on the host, using multi-pass rendering techniques. For each ray launch pass, per-ray intermediate results are fetched from accumulation buffer data structures in GPU global memory, accumulated with new results, and stored back to GPU global memory in preparation for return or for subsequent ray launches. Intermediate color results from shading and the internal state of random number generators are the key data structures that are maintained between ray launches. Using these techniques it was possible to render nested transparent molecular surfaces with secondary ray depths of up to 20 levels of recursion without encountering GPU kernel timeouts. The current implementation allows control over the number of ambient occlusion samples computed "per-hit", within the same ray launch pass. Currently, the best ambient occlusion performance is usually obtained by maximizing the ratio of per-hit ambient occlusion samples to per-pixel antialiasing samples, up to 


\begin{tabular}{|c|c|c|c|c|c|c|c|}
\hline \multirow[b]{2}{*}{ Movie Resolution } & \multirow{2}{*}{$\begin{array}{c}\text { Rendering Mode } \\
\text { Ambient Occlusion (AO) Parameters }\end{array}$} & \multirow[b]{2}{*}{ Node Type } & \multirow[b]{2}{*}{ Nodes } & \multicolumn{4}{|c|}{ Full-Movie Wall Clock Execution Time } \\
\hline & & & & $\begin{array}{c}\text { Script } \\
\text { Loading } \\
\end{array}$ & $\begin{array}{c}\text { State } \\
\text { Loading }\end{array}$ & $\begin{array}{l}\text { Geometry and } \\
\text { Rendering }\end{array}$ & Total \\
\hline \multirow{2}{*}{$\begin{array}{l}\text { "Presentation" } \\
1057 \times 652 \\
689,164 \text { pixels }\end{array}$} & $\begin{array}{l}\text { TachyonL-OptiX GPU ray tracing } \\
12 \text { samples/pixel, } 12 \text { AO samples/hit }\end{array}$ & $\begin{array}{l}\text { XK7 } \\
\text { XK7 } \\
\text { XK7 } \\
\text { XK7 }\end{array}$ & $\begin{array}{c}64 \\
128 \\
256 \\
512\end{array}$ & $\begin{array}{l}2 \mathrm{~s} \\
2 \mathrm{~s} \\
4 \mathrm{~s} \\
9 \mathrm{~s}\end{array}$ & $\begin{array}{c}38 \mathrm{~s} \\
60 \mathrm{~s} \\
121 \mathrm{~s} \\
195 \mathrm{~s}\end{array}$ & $\begin{array}{l}213 \mathrm{~s} \\
109 \mathrm{~s} \\
61 \mathrm{~s} \\
30 \mathrm{~s}\end{array}$ & $\begin{array}{l}253 \mathrm{~s} \\
171 \mathrm{~s} \\
186 \mathrm{~s} \\
234 \mathrm{~s}\end{array}$ \\
\hline & $\begin{array}{c}\text { Tachyon CPU ray tracing } \\
12 \text { samples/pixel, } 12 \text { AO samples/hit }\end{array}$ & $\begin{array}{l}\text { XE6 } \\
\text { XE6 } \\
\text { XE6 } \\
\end{array}$ & $\begin{array}{l}128 \\
256 \\
512 \\
\end{array}$ & $\begin{array}{l}2 \mathrm{~s} \\
4 \mathrm{~s} \\
7 \mathrm{~s} \\
\end{array}$ & $\begin{array}{c}83 \mathrm{~s} \\
125 \mathrm{~s} \\
221 \mathrm{~s} \\
\end{array}$ & $\begin{array}{l}943 \mathrm{~s} \\
560 \mathrm{~s} \\
330 \mathrm{~s} \\
\end{array}$ & $\begin{array}{c}1,029 \mathrm{~s} \\
692 \mathrm{~s} \\
560 \mathrm{~s} \\
\end{array}$ \\
\hline \multirow{2}{*}{$\begin{array}{c}\text { HD } \\
1920 \times 1080 \\
2,073,600 \text { pixels }\end{array}$} & $\begin{array}{l}\text { TachyonL-OptiX GPU ray tracing } \\
144 \text { samples/pixel, } 1 \text { AO sample/hit } \\
\text { (avoids GPU kernel timeout) }\end{array}$ & $\begin{array}{l}\text { XK7 } \\
\text { XK7 } \\
\text { XK7 } \\
\text { XK7 }\end{array}$ & $\begin{array}{c}64 \\
128 \\
256 \\
512\end{array}$ & $\begin{array}{c}2 \mathrm{~s} \\
4 \mathrm{~s} \\
7 \mathrm{~s} \\
14 \mathrm{~s}\end{array}$ & $\begin{array}{c}38 \mathrm{~s} \\
74 \mathrm{~s} \\
110 \mathrm{~s} \\
184 \mathrm{~s}\end{array}$ & $\begin{array}{l}655 \mathrm{~s} \\
331 \mathrm{~s} \\
171 \mathrm{~s} \\
125 \mathrm{~s}\end{array}$ & $\begin{array}{l}695 \mathrm{~s} \\
410 \mathrm{~s} \\
288 \mathrm{~s} \\
325 \mathrm{~s}\end{array}$ \\
\hline & $\begin{array}{c}\text { Tachyon CPU ray tracing } \\
12 \text { samples/pixel, } 12 \text { AO samples/hit }\end{array}$ & $\begin{array}{l}\mathrm{XE6} \\
\mathrm{XE} 6\end{array}$ & $\begin{array}{l}256 \\
512\end{array}$ & $\begin{array}{c}7 \mathrm{~s} \\
13 \mathrm{~s}\end{array}$ & $\begin{array}{l}160 \mathrm{~s} \\
211 \mathrm{~s}\end{array}$ & $\begin{array}{c}1,374 \mathrm{~s} \\
808 \mathrm{~s}\end{array}$ & $\begin{array}{l}1,541 \mathrm{~s} \\
1,032 \mathrm{~s}\end{array}$ \\
\hline
\end{tabular}

Table 3: VMD Parallel Movie Rendering Performance Tests: 1,079 Frame HIV-1 Movie.

the point of incurring GPU kernel timeouts.

\section{PERFORMANCE RESULTS}

Performance data were collected for renderings performed using a multi-core CPU version of the Tachyon ray tracing engine running on the Opteron CPUs of the Cray XE6 and XK7 compute nodes, labeled "Tachyon". We developed a new ray tracing engine, a subset or "light" version of Tachyon, that has been rewritten for GPU accelerated execution on the Cray XK7 nodes using a combination of CUDA and OptiX, which we have labeled "TachyonL-OptiX".

The performance of VMD single-frame rendering was evaluated on Blue Waters and desktop workstations for a 2.4 million atom chromatophore model [23] using ray tracing techniques, including antialiasing (48 samples/pixel), shadows, and ambient occlusion (AO) lighting (3 samples/hit), depth cueing, transmission rays, and angle-modulated transparency for a $1024 \times 1024$ pixel image with 14 cylinders, 18 spheres, and 4,756,308 triangles. The predominantly transparent geometry is a challenging ray tracing stress test because it creates a massive workload in the form of secondary rays. Table 1 presents performance results for varying node types and rendering techniques. The "Loading, Geometry Calc." step, which includes disk I/O, script execution, and molecular surface calculation time, also benefits from GPU acceleration. 'Xeon E5' is a 16-core Xeon E5-2687W; 'Xeon E5 GPU' is an NVIDIA Quadro K6000; 'Xeon X5550' is a 16-core Xeon X5550; 'Xeon X5550 Two GPUs' are two NVIDIA Tesla K20c GPUs. For 'XK7/No GPU', testing was done with explicitly disabled GPUs.

Table 2 presents ray tracing performance on an NVIDIA Quadro K6000 GPU, with different rendering features enabled. For all test cases, preparing the OptiX context took $\approx 0.367 \mathrm{~s}$, building the OptiX "MedianBvh" acceleration structure took $\approx 1.447 \mathrm{~s}$, and file $\mathrm{I} / \mathrm{O}$ took $\approx 0.007 \mathrm{~s}$. The number of antialiasing samples (rays averaged) per pixel is a strongly multiplicative factor on the overall ray tracing workload because it also increases the overall number of secondary rays generated for shadows, ambient occlusion lighting, etc. Increasing the number of samples per pixel from 1 to 12 increases overall ray tracing time by $\approx 6.4 \times$. The addition of shadows from direct lighting increases runtime by a factor of $\approx 5$ - $6 \times$ over antialiasing alone (also increasing with maximum ray recursion depth), and enabling ambient occlusion with a sufficient number of samples to achieve ex- cellent quality increases runtime by another $\approx 20.5 \times$. The VMD OpenGL GLSL rendering time for the same test scene with only direct lighting, and a limited transparent surface rendering scheme was under $90 \mathrm{~ms}$.

VMD parallel movie rendering performance was evaluated on Blue Waters for a 1,079 frame movie of the allatom HIV-1 capsid [27] using ray tracing techniques, including antialiasing, shadows and ambient occlusion lighting, depth cueing, transmission rays, and angle-modulated transparency. Table 3 includes results for varying movie resolutions, rendering engine, node types, and node counts. Work is scheduled over all nodes with a dynamic load balancer assigning individual frames to nodes, thereby ameliorating the impact of large (up to one order-of-magnitude) variations in per-frame geometric computation and rendering times throughout the process of movie rendering [20]. The individual frame rendering times for HIV-1 are much shorter than (less than $10 \%$ of) the chromatophore test case shown in Tables 1 and 2 because the HIV-1 scene creates far fewer secondary rays associated with transmission rays through transparent surfaces, and for shadows and ambient occlusion lighting. We note that the I/O runtime associated with initial loading of common structure data increases with the number of compute nodes in use, which is a result of many nodes competing while reading the same files [20].

\section{CONCLUSIONS AND FUTURE WORK}

The new GPU-accelerated molecular surface algorithm and GPU-accelerated ray tracing engine have significantly improved the performance of photorealistic VMD movie renderings, particularly for molecular scenes with large amounts of transparent geometry, and ambient occlusion lighting. We expect that continued algorithm development and performance tuning will increase GPU ray tracing performance further and will completely eliminate GPU kernel timeout concerns, even for highly complex molecular scenes. The performance increases provided by GPUs are large enough that several of the movie rendering cases presented demonstrate I/O bound behavior at high node counts, when loading common structural information on all nodes. We plan to address this I/O performance limitation by broadcasting common molecular data to all nodes using MPI. A currently unexplored avenue for reduction of I/O activity and reduced movie generation turnaround times relates to onthe-fly movie compression. With such an approach, the 
Tesla K20X GPUs within each Cray XK7 node could be used to accelerate H.264 video encoding and decoding, enabling compressed movie renderings to be streamed to disk using only a fraction of the storage and network bandwidth that is required for conventional image-at-a-time output storage.

\section{ACKNOWLEDGMENTS}

This research is part of the Blue Waters sustained-petascale computing project supported by NSF award OCI 07-25070, and "The Computational Microscope" NSF PRAC award. Blue Waters is a joint effort of the University of Illinois at Urbana-Champaign and its National Center for Supercomputing Applications (NCSA). The authors wish to acknowledge support of the CUDA Center of Excellence at the University of Illinois, and NIH funding through grants 9P41GM104601 and 5R01GM098243-02.

\section{REFERENCES}

[1] J. F. Blinn. A Generalization of Algebraic Surface Drawing. ACM Transactions on Graphics, 1:235-256, 1982.

[2] M. L. Connolly. Analytical molecular surface calculation. Journal of Applied Crystallography, 16:548-558, 1983.

[3] D. D'Agostino, A. Clematis, S. Decherchi, W. Rocchia, L. Milanesi, and I. Merelli. CUDA accelerated molecular surface generation. Concurrency and Computation: Practice and Experience, 2013.

[4] S. Decherchi and W. Rocchia. A general and robust ray-casting-based algorithm for triangulating surfaces at the nanoscale. PLoS ONE, 8(4):e59744, 042013.

[5] J. Enos, C. Steffen, J. Fullop, M. Showerman, G. Shi, K. Esler, V. Kindratenko, J. E. Stone, and J. C. Phillips. Quantifying the impact of GPUs on performance and energy efficiency in HPC clusters. In International Conference on Green Computing, pages 317-324, 2010.

[6] W. Humphrey, A. Dalke, and K. Schulten. VMD Visual Molecular Dynamics. J. Mol. Graphics, 14:33-38, 1996.

[7] Y. Kanamori, Z. Szego, and T. Nishita. GPU-based fast ray casting for a large number of metaballs. Computer Graphics Forum, 27(3):351-360, 2008.

[8] M. Krone, S. Grottel, and T. Ertl. Parallel Contour-Buildup Algorithm for the Molecular Surface. In Proceedings of IEEE Symposium on Biological Data Visualization, 2011.

[9] M. Krone, J. E. Stone, T. Ertl, and K. Schulten. Fast visualization of Gaussian density surfaces for molecular dynamics and particle system trajectories. In Euro Vis - Short Papers 2012, pages 67-71, 2012.

[10] E. H. Lee, J. Hsin, M. Sotomayor, G. Comellas, and K. Schulten. Discovery through the computational microscope. Structure, 17:1295-1306, 2009.

[11] B. G. Levine, J. E. Stone, and A. Kohlmeyer. Fast analysis of molecular dynamics trajectories with graphics processing units-radial distribution function histogramming. J. Comp. Phys., 230:3556-3569, 2011.

[12] N. Lindow, D. Baum, S. Prohaska, and H.-C. Hege. Accelerated visualization of dynamic molecular surfaces. Computer Graphics Forum, 29:943-952, 2010.
[13] C. Müller, S. Grottel, and T. Ertl. Image-Space GPU Metaballs for Time-Dependent Particle Data Sets. In Proceedings of Vision, Modelling and Visualization ( $V M V$ 'O7), pages 31-40, 2007.

[14] S. G. Parker, J. Bigler, A. Dietrich, H. Friedrich, J. Hoberock, D. Luebke, D. McAllister, M. McGuire, K. Morley, A. Robison, and M. Stich. OptiX: a general purpose ray tracing engine. In ACM SIGGRAPH 2010 papers, SIGGRAPH '10, pages 66:1-66:13, New York, NY, USA, 2010. ACM.

[15] K. Reda, A. Knoll, K. Nomura, M. E. Papka, A. E. Johnson, and J. Leigh. Visualizing large-scale atomistic simulations in ultra-resolution immersive environments. IEEE Symposium on Large-Scale Data Analysis and Visualization, 2013.

[16] F. M. Richards. Areas, volumes, packing, and protein structure. Annual Review of Biophysics and Bioengineering, 6(1):151-176, 1977.

[17] E. Roberts, J. E. Stone, and Z. Luthey-Schulten. Lattice microbes: High-performance stochastic simulation method for the reaction-diffusion master equation. J. Comp. Chem., 34:245-255, 2013.

[18] J. E. Stone. An Efficient Library for Parallel Ray Tracing and Animation. Master's thesis, Computer Science Department, University of Missouri-Rolla, April 1998.

[19] J. E. Stone, D. J. Hardy, I. S. Ufimtsev, and K. Schulten. GPU-accelerated molecular modeling coming of age. J. Mol. Graph. Model., 29:116-125, 2010 .

[20] J. E. Stone, B. Isralewitz, and K. Schulten. Early experiences scaling VMD molecular visualization and analysis jobs on Blue Waters. Proceedings of the Extreme Scaling Workshop, 2013.

[21] J. E. Stone, A. Kohlmeyer, K. L. Vandivort, and K. Schulten. Immersive molecular visualization and interactive modeling with commodity hardware. Lect. Notes in Comp. Sci., 6454:382-393, 2010.

[22] J. E. Stone, J. C. Phillips, P. L. Freddolino, D. J. Hardy, L. G. Trabuco, and K. Schulten. Accelerating molecular modeling applications with graphics processors. J. Comp. Chem., 28:2618-2640, 2007.

[23] J. Strumpfer, M. Sener, and K. Schulten. How quantum coherence assists photosynthetic light harvesting. J. Phys. Chem. Lett., 3:536-542, 2012.

[24] M. Totrov and R. Abagyan. The contour-buildup algorithm to calculate the analytical molecular surface. Journal of Structural Biology, 116:138-143, 1995.

[25] L. G. Trabuco, E. Villa, K. Mitra, J. Frank, and K. Schulten. Flexible fitting of atomic structures into electron microscopy maps using molecular dynamics. Structure, 16:673-683, 2008.

[26] H. Yu, C. Wang, R. W. Grout, J. H. Chen, and K.-L. Ma. In situ visualization for large-scale combustion simulations. Computer Graphics and Applications, IEEE, 30(3):45-57, 2010.

[27] G. Zhao, J. R. Perilla, E. L. Yufenyuy, X. Meng, B. Chen, J. Ning, J. Ahn, A. M. Gronenborn, K. Schulten, C. Aiken, and P. Zhang. Mature HIV-1 capsid structure by cryo-electron microscopy and all-atom molecular dynamics. Nature, 497:643-646, 2013. 